\title{
ENVELOPE AND MULTI-SLIT EMITTANCE MEASUREMENTS AT FERMILAB A0-PHOTOINJECTOR AND COMPARISON WITH SIMULATIONS *
}

\author{
C.M. Bhat $^{\dagger}$, J.-P Carneiro ${ }^{\ddagger}$, R.P. Fliller, G. Kazakevich, J. Ruan, J. Santucci \\ FNAL, Batavia, IL 60510, USA
}

\section{Abstract}

Recently we have measured the envelope and the transverse emittance of an $0.85 \mathrm{nC}$ electron beam at the Fermilab A0-Photoinjector facility. The transverse emittance measurement was performed using the multi-slit method. The data have been taken with an unstacked 2.8 ps laser pulse. In this paper we report on these beam measurements and compare the results with the predictions from beam dynamics codes ASTRA and GPT using 3D space charge routines.

\section{INTRODUCTION}

A layout of the A0 Photoinjector currently installed in the A0 hall at Fermilab is presented in Figure 1. It consists of an RF-gun resonating in the $\mathrm{TM}_{010, \pi}$ mode at $1.3 \mathrm{GHz}$ and followed by a 9-cell superconducting ILC-type cavity. A cesium telluride $\left(\mathrm{Cs}_{2} \mathrm{Te}\right)$ photocathode (fabricated and rejuvenated on-site in a photo-cathode preparation chamber) allows the generation of electron bunches of up to several nano-coulombs when illuminated by a pulsed UV laser $(\lambda=263 \mathrm{~nm})$. Three solenoids (a primary, a secondary and a bucking that allows the cancellation of the magnetic field on the photo-cathode) focus the space charge dominated beam prior acceleration by the 9 -cell cavity.

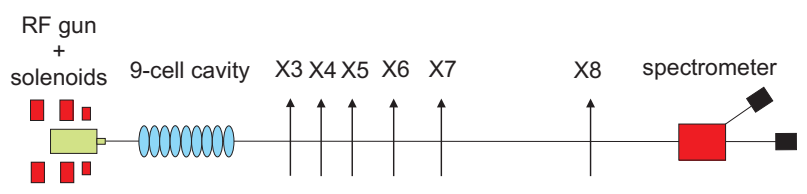

Figure 1: Layout of the A0 photoinjector.

The beam is transported up to a spectrometer by several quadrupoles. The distance between the photo-cathode and the spectrometer dipole is in the order of $\sim 11$ meters. As depicted in Figure 1, several screens and multi-slit emittance probes are located in the transport line for diagnostic purposes. For the measurements presented in this paper, the measured longitudinal length of UV laser pulses is $\sigma_{Z} \simeq 2.8 \mathrm{ps}$ (RMS gaussian) for a transverse size supposed uniform with a radius of $1.2 \mathrm{~mm}$. The electron bunch charge is $Q=0.85 \mathrm{nC}$. The peak field on the RF gun is 35.2

\footnotetext{
* Work supported by Fermi National Accelerator Laboratory operated by Fermi Research Alliance, LLC under Contract No. DE-AC0207CH11359 with the United States Department of Energy.

†cbhat@fnal.gov

† carneiro@fnal.gov
}

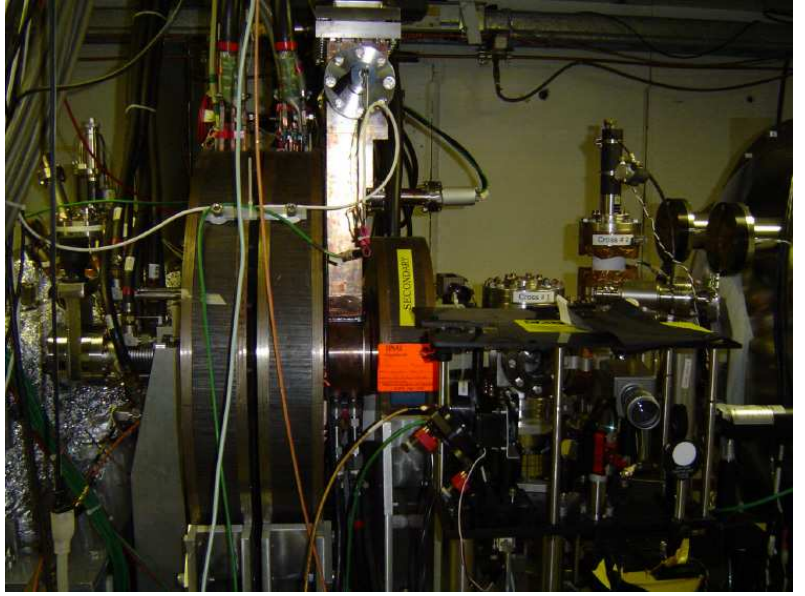

Figure 2: Fermilab RF gun as installed at the A0Photoinjector facility.

$\mathrm{MV} / \mathrm{m}$ and the accelerating field on the 9-cell cavity in the order of $11.8 \mathrm{MV} / \mathrm{m}$. The three solenoids are set to $270 \mathrm{~A}$ each which produces a longitudinal peak magnetic field of $0.1354 \mathrm{~T}$. The launch phase of the RF gun is about $40^{\circ}$ and the 9 -cell phase set to minimize the energy spread. The measured kinetic energy of the beam after full acceleration was $\sim 15.3 \mathrm{MeV}$. A picture of the RF gun and the three solenoids as installed in the A0 hall at Fermilab is presented in Figure 2.

\section{CODE DESCRIPTIONS}

Two tracking codes are used in this paper to simulate the beam dynamics in the photo-injector : ASTRA and General Particle Tracer GPT. ASTRA has been developed by DESY (Hamburg, Germany) and is mainly used for the design of electron photo-injectors. GPT is a well established simulation code developed by Pulsar Physics (The Netherlands).

Both ASTRA and GPT perform the integration of the relativistic equations of motion by the Runge-Kutta method of fourth order for ASTRA with a fixed integration time step and fifth order for GPT with an adaptive time step. The electromagnetic fields include external contribution from accelerating and focusing fields and internal from the beam space charge forces. Both codes have the ability of using 2D external electric fields defined on the cavity axis or 3D external electric fields defined on a rectangular mesh. GPT can handle both 2D or 3D external solenoid magnetic fields, while ASTRA only supports $2 \mathrm{D}$ ones. For the simu- 
lations presented in this paper, both codes have been provided with the exact $2 \mathrm{D}$ electric fields on axis for the RF gun and the 9-cell cavity and with the exact 2D magnetic fields on axis for the solenoids. The electric fields have been simulated with the code CST MicroWave Studio [3] and the magnetic field with the code POISSON [4].

ASTRA and GPT support 2D or 3D space charge calculations. For the simulations presented in this paper, the space-charge forces have been computed using the 3D space charge routines. For both codes, the space-charge fields are calculated in the beam rest frame via Poisson's equation :

$$
\nabla^{2} \phi(r, \theta, z)=-\frac{\rho(r, \theta, z)}{\epsilon_{0}}
$$

with $\phi$ the electrostatic potential, $\rho$ the charge density, $\epsilon_{0}$ the dielectric constant and Lorentz's transformed back into the laboratory frame. A 3D cartesian grid is used for both codes with the calculation of the electrostatic potential at each grid points. The ASTRA simulations presented in this paper were performed with $10^{4}$ macro-particles and a mesh of $64 \times 64 \times 64$ while the GPT simulations used $5 \cdot 10^{4}$ macro-particles and a mesh of $20 \times 20 \times 20$.

\section{ENERGY SIMULATIONS}

Figure 3 presents ASTRA and GPT simulations for the kinetic energy of a $Q=0.85 \mathrm{nC}$ bunch along the beamline, using parameters of our experimental setup as presented earlier. An excellent agreement is observed between these codes. Nevertheless, the simulated kinetic energy ( 16 $\mathrm{MeV})$ is slightly higher than the measured one (15.3 MeV).

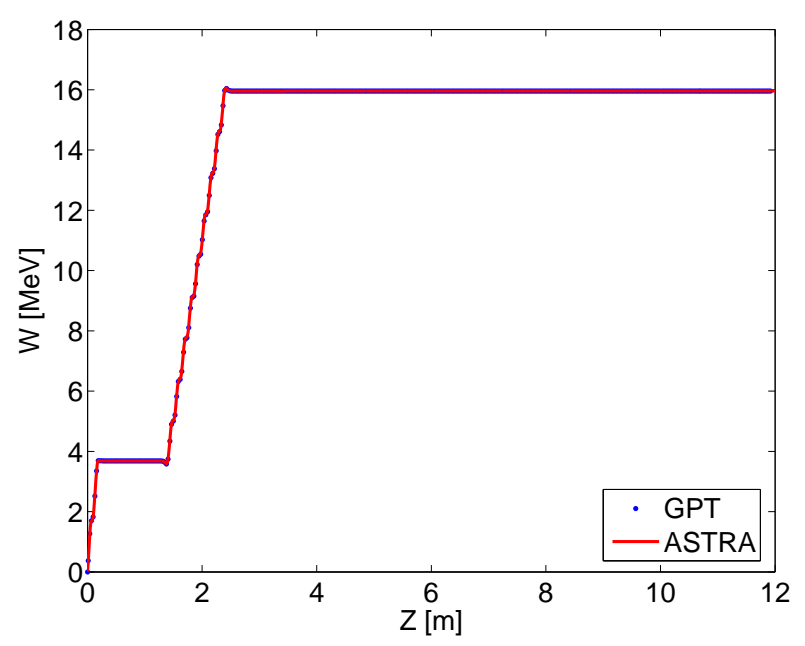

Figure 3: Kinetic energy along the beamline from ASTRA and GPT.

\section{BEAM ENVELOPE MEASUREMENTS}

Figure 4 presents the measured horizontal and vertical beam sizes at different locations along the beamline and a comparison with the ASTRA and GPT. The beam envelop measurements have been carried out for $Q=0.85 \mathrm{nC} / \mathrm{pulse}$ (with a typical pulse-to-pulse charge variation of about 5\%) after de-gaussing and turning off all of the magnetic elements between X3 and X8 (see Figure 1) and successfully transporting the beam from the source to the spectrometer dump.

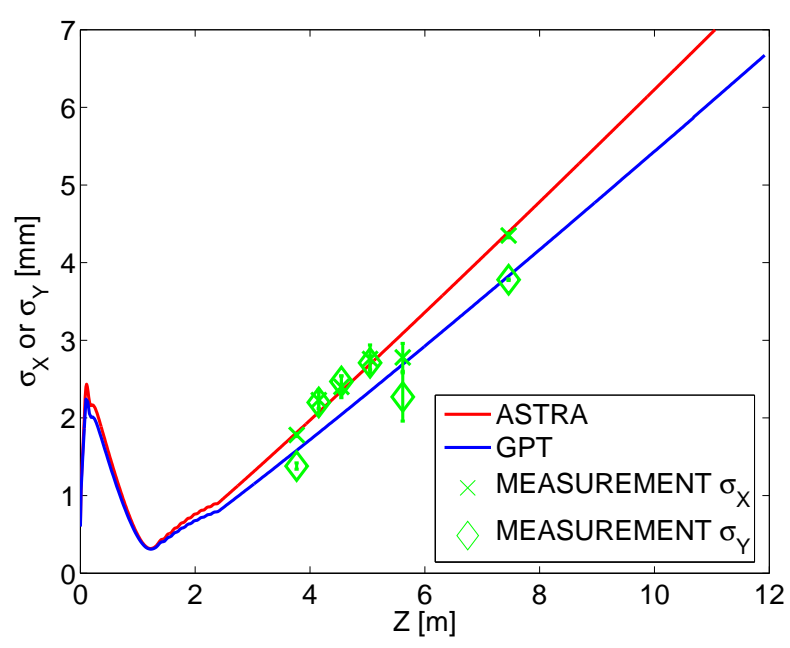

Figure 4: Beam envelope measurements and comparison with the simulation codes ASTRA and GPT for $Q=0.85 \mathrm{nC}$.

Typical beam images taken by CCD cameras at various locations in the beam-line are shown in Figure 5.
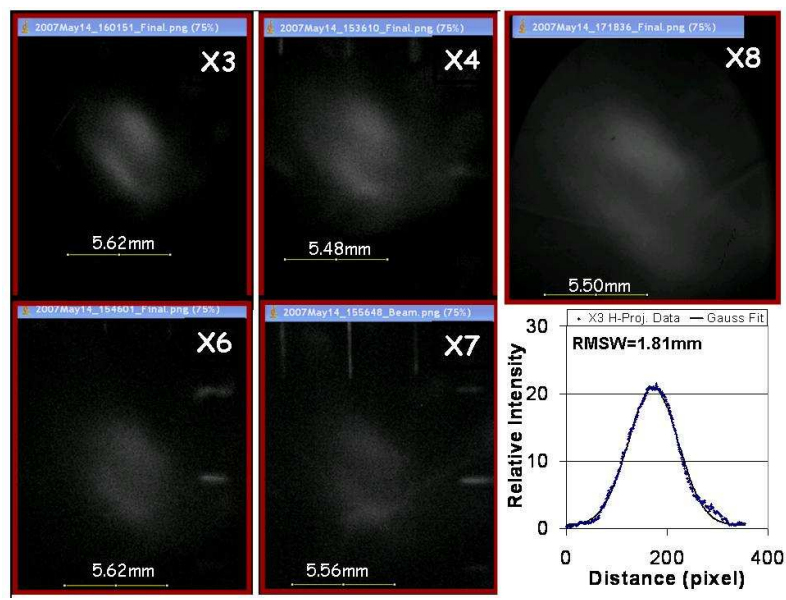

Figure 5: Typical beam envelopes measured at X3, X4, X6, $\mathrm{X} 7$ and $\mathrm{X} 8$. Horizontal projection data with gaussian fit for the data corresponding to $\mathrm{X} 3$ is also shown.

OTR screens were used at locations X3, X4, X6 and X7 with trains of 10 pulses and YAG screens at X5,X8 with a single pulse. The horizontal and vertical RMS beam spot size were extracted from each image by a Gaussian fit of the projected data (as displayed in Figure 5). For each measurement, a statistical mean and average deviation on 3 images were calculated. A reasonably good agreement is presented in Figure 4 between the measurement of the transverse spot sizes at different locations along the beam- 
line and the codes ASTRA and GPT. As expected, the asymmetry observed during the measurement was not reported by the simulation codes since a symmetric laser pulse (radius of $1.2 \mathrm{~mm}$ ) was chosen for both codes. The next step in the simulation would be to implement a realistic transverse and longitudinal laser pulse (taking into account the asymmetries and inhomogeneities of the laser pulse).

\section{BEAM EMITTANCE MEASUREMENT}

The emittance measurements were done using the slit technique: two actuator-mounted slit masks were used to measure the horizontal and vertical emittances $\left(\epsilon_{x}\right.$ and $\left.\epsilon_{y}\right)$ at $z \simeq 5.6 \mathrm{~m}$ (X7, see Figure 1). The beam-lets passing through the slits are viewed with a YAG screen located at a distance $d=1844 \mathrm{~mm}$ from the slits (X8, see Figure 1). The masks consist of $6 \mathrm{~mm}$ thick tungsten slats with 50 $\mu \mathrm{m}$ wide slits spaced $1 \mathrm{~mm}$ apart. The RMS normalized emittance was computed using the relation [5] :

$$
\epsilon_{n, r m s}=\beta \gamma \sqrt{\left\langle u^{2}\right\rangle\left\langle\left(u^{\prime}\right)^{2}\right\rangle-\left\langle u u^{\prime}\right\rangle^{2}},
$$

where $\beta c$ is the velocity of the beam, $\gamma$ is the Lorentz factor, $u$ and $u^{\prime}$ are the transverse coordinate and divergence of $x$ or $y$, and \langle\rangle denotes an RMS value. We adopted the formalisum explained in ref [6] to extract the un-correlated emittance as defined by Eq. 2 .
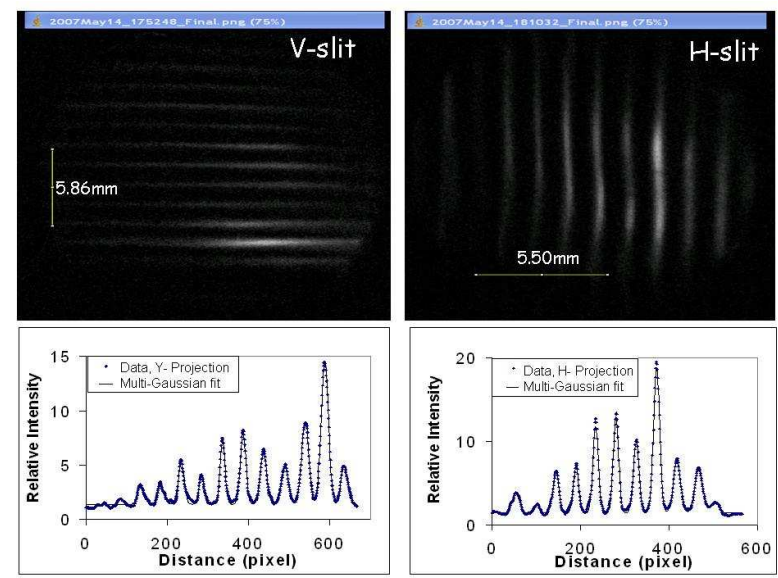

Figure 6: Measured beam-lets at X8 (mult-slits at X7). Projection data with multi-gaussian fits are shown below.

Figure 6 shows typical beam-let data used to measure the horizontal and vertical emittances at location X7. The corresponding ASTRA and GPT predictions on the emittances are shown in Figure 7. We find that there are considerable differences between the models' predictions and the measured emittances. This may be primarily due to significant asymmetry seen in the beam; the simulation assumed that the beam profiles were symmetric gaussians in the X7 location.

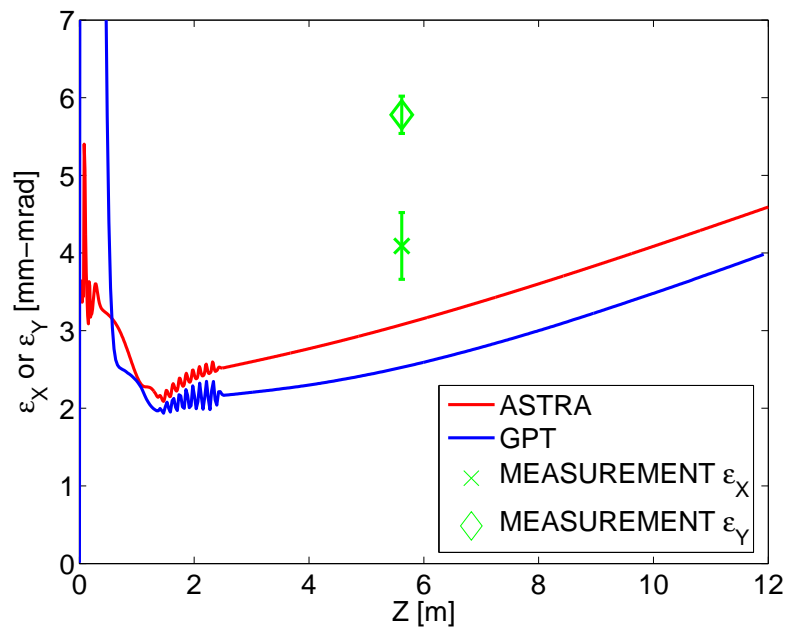

Figure 7: Comparison between measured and predicted transverse emittances at $\mathrm{X} 7$.

\section{CONCLUSION}

We have carried out model predictions for A0Photoinjector beam transport using the codes ASTRA and GPT for $Q=0.85 \mathrm{nC}$ beam including space-charge effects. There is quite good agreement between predictions from these two codes. These predictions have been compared with the measured beam envelopes at various locations in the beamline and the beam emittances from the multi-slit technique. Good agreement is seen between envelope-data and the model calculations. However, significant differences are observed between multi-slit data and the models. The sources for the differences are being investigated.

\section{ACKNOWLEDGMENT}

The authors would like to thank P. Piot for his help in the simulations using GPT. We are indebted to V. Scarpine for his help in data acquisition system at the A0-photoinjector. Our special thanks are due to H. Edwards and D. Edwards for many useful disscussions.

\section{REFERENCES}

[1] K. Flöttmann, ASTRA user manual available at : http://www.desy.de/ mpyflo/Astra_dokumentation/

[2] GPT User Manual, Pulsar Physics, De Bongert 23, Soest, The Netherlands, http://www.pulsar.nl/gpt/.

[3] CST MicroWave Studio, Version 4.0, Darmstadt, 2002.

[4] POISSON/SUPERFISH group of codes, Version 4.28 for PC, Los Alamos, 2002.

[5] P.M. lapostolle, IEEE Trans. Nucl. Sci., Vol 18, N 3, pp. 1101-1104, June 1971.

[6] C. Lejeune and J. Aubert "Emittance and Brightness: Definitions and Measurements" Adv. in Electronics and Electron Phys, Suppl. 13A, Page 159(1980); M. Zhang, FERMILABTM-1988 (1996). 